\title{
Electronic Case Reporting of STls: Assessing EHR Generated CCDs
}

\author{
Kim Peifer ${ }^{\star 1}$, Mary Stark ${ }^{2}$, Rita Altamore ${ }^{1}$ and Julieann Simon ${ }^{1}$ \\ ${ }^{1}$ Washington State Department of Health, Shoreline, WA, USA; ${ }^{2}$ Planned Parenthood of the Great Northwest and the Hawaiian \\ Islands, Boise, ID, USA
}

\section{Objective}

We reviewed CCDs (a type of consolidated clinical data architecture (C-CDA) document) shared by our clinical partner, Planned Parenthood of the Great Northwest and Hawaiian Islands (PPGNHI) since October, 2015. Analyses focuses on:

-Completeness

-Degree to which the CCD matches program area information needs

-Differences in EHR generation methods

-Presence and location of triggers (based on the Reportable Conditions Trigger Codes) that would initiate CCD generation.

\section{Introduction}

Under the CDC STD Surveillance Network (SSuN) Part B grant, WA $\mathrm{DOH}$ is testing electronic case reporting $(\mathrm{eCR})$ of sexually transmitted infections (STI) from a clinical partner.

\section{Methods}

Two methods of CCD generation, based on existing EHR capabilities, were used to create CCDs that were delivered to WA DOH using secure file transport protocol (SFTP). PPGNHI uses the NextGen EHR system.

The first batch received was extracted using the Medical Summary Utility. Random selection of cases (25) from lab positive Chlamydia (CT), Gonorrhea (GC) or Syphilis encounters with a follow-up plan in the EHR (1/1/2015-3/31/2015). Each CCD contained manually selected encounters (related to STI case).

Cases are now extracted directly from a patient chart (File-->Generate CCD). Two types of CCDs can be created: single encounter CCDs and longitudinal encounter CCDs.

The CCDs were analyzed for completeness, crossover with the existing paper case report, and with relevant CDA and C-CDA Implementation Guide (IG) standards.

\section{Results}

This analysis includes four reportable events across 6 CCDs. One event is represented by both a longitudinal CCD and 2 single encounter CCDs.

The CCDs contained most of the basic demographic information requested in the paper case report with the exception of "middle initial".

Information on the important paper case report components "gender of sex partner" and "partner management plan" are not found in the CCD.

The CCD Results section contained lab tests and results that include site of infection and could confirm diagnosis. The ordered test (panel) is not coded, though the individual tests performed are LOINC coded.

The CCD Medications section meets STI program needs for information about treatment in a case report. Information is represented using RxNorm codes as specified by the C-CDA IG.

The CCD Problems section was not present in documents generated using the MSU but was present in documents created using File --> Generate CCD from the patient chart. The Problems section and coded entries (ICD-9-CM and ICD-10) are required for CCDs. The
Problems do not include effective dates, which are not required by the IG.

Pregnancy status, and information about HIV testing (including previous positive), are present in the CCD Problems section only if the encounter during which testing occurred is included in the CCD submitted.

Using the CCD in place of the paper case report requires understanding of the clinical workflow and use of EHR. Two instances that require specific attention are the "exposure" status of the case (known/possibly exposed vs. not shared/not known), and the "presentation" of the diagnosis (symptomatic vs asymptomatic). For example, the ICD-10 code Z11.3 (encounter for screening for infections with a predominantly sexual mode of transmission), cannot be interpreted as a true "screening", as this diagnosis is recorded for all visits that include STI testing. Similarly, a code for exposure to STIs is sometimes used, but not consistently enough to allow reliable identification of cases in which the patient was tested due to an exposure or possible exposure. Work with our clinical partner to understand what inferences can and should be made is an important part of evaluating the $\mathrm{CCD}$ as a replacement to the paper case report.

\section{Conclusions}

The CCDs submitted to DOH show that most information requested in an STI case report can be found in a CCD with some exceptions, notably "gender of sex partners" and "partner management plan". Some information is only inconsistently present, for example, exposure status and presentation.

Understanding how the CCD could replace the paper case report requires working with the reporter to insure that the information is interpreted on the receiving end in the same way that it is interpreted in the clinical workflow and entered in the EHR.

\section{Keywords}

electronic case reporting; informatics; data sharing; electronic health records; sexually transmitted diseases

\section{Acknowledgments}

-Funding for this project in part provided by the U.S. Centers for Disease Control and Prevention, STD Surveillance Network, PS13-1306

-This study/report was supported in part by an appointment to the Applied Public Health Informatics Fellowship and the Informatics Training in Place Program administered by CSTE and funded by the Centers for Disease Control and Prevention (CDC) Cooperative Agreement 3U38-OT000143-01S3.

-Washington DOH SSuN Part B team, especially Rita Altamore, Julie Simon, and Teal Bell

-Planned Parenthood of the Great Northwest and Hawaiian Islands, especially Mary Stark and Dr. Laurel Kuehl

\section{*Kim Peifer}

E-mail: kim.peifer@doh.wa.gov 